\title{
Keeping our eyecare providers and patients safe during the COVID- 19 pandemic
}

\author{
Kelvin Yi Chong Teo ${ }^{1,2,3} \cdot$ R. V. Paul Chan $\mathbb{1}^{4} \cdot$ Chui Ming Gemmy Cheung $\mathbb{( i}^{1,2}$
}

Received: 30 April 2020 / Revised: 1 May 2020 / Accepted: 1 May 2020 / Published online: 14 May 2020

(c) The Royal College of Ophthalmologists 2020

The coronavirus disease 2019 (COVID-19) pandemic caused by the severe acute respiratory syndrome coronavirus 2 (SARS-CoV-2) is the defining event of this generation. To date, there are over 2 million cases including more than 145,000 deaths with this number constantly rises day by day. The lack of a cure or a viable vaccine has resulted in the implementation of extreme social distancing measures with lockdowns declared in many countries to minimise person-to-person interactions to prevent the transmission of the virus [1]. These measures have a profound impact on health care provision. A few subspecialities such as ENT, anaesthesia and ophthalmology are considered to be at higher risk than others due to the close proximity to patients and the nature of procedures associated with higher transmission risk [2-5].

Chandra et al. [6] summarises the latest evidence related to the mode of transmission of COVID-19 and current recommendations for the safe practice of ophthalmology. This article is timely and highlights several key areas.

First, during the acute crisis, understanding the mode(s) of transmission is essential to guide our policy makers and healthcare workers regarding the appropriate measures to protect patients and frontliners (https://www.rcophth.ac.uk/ 2020/04/covid-19-update-and-resources-for-ophthalmologists/). However, there remain differences in recommendations towards what is considered the appropriate level of personal protective equipment (PPE) for ophthalmology procedures. For

\section{Chui Ming Gemmy Cheung}

gemmy.cheung.c.m@snec.com.sg

1 Singapore Eye Research Institute, Singapore National Eye Centre, Singapore, Singapore

2 Duke-NUS Medical School, National University of Singapore, Singapore, Singapore

3 Save Sight Institute, University of Sydney, Sydney, Australia

4 Department of Ophthalmology and Visual Sciences, Illinois Eye and Ear Infirmary, University of Illinois at Chicago, Chicago, USA example, whether N95 or surgical masks are adequate protection for outpatient-based intravitreal injection remains unclear. One reason for such variation may be related to the difference in rates of community spread in different countries. In some parts of Asia, like Singapore, where community spread is relatively low, the risk of an asymptomatic patient with no contact history harbouring undiagnosed COVID-19 infection is low. On the other hand, in settings where community infection rate is high, it may be advisable to treat every patient as a potential infected case. Similar variations also exist regarding whether pre-op testing for COVID-19 should be conducted in patients scheduled for surgery and even the timing of pre-op testing (same day or 48-72 $\mathrm{h}$ prior to the surgery) is uncertain. Recommendations for surgical PPE is also variable and there is no consensus on whether N95 mask should be donned during procedures such as phacoemulsification and vitrectomy. Hence each country and healthcare system will need to weigh up the overall transmission risk and resource management of PPE to inform the guidelines for ophthalmology practices. With increasing efforts to perform community testing in many countries, current recommendations can be modified to adapt to the needs for individual settings.

The ophthalmology community as a whole will have to face major modifications to how we manage outpatient and office-based visits during and after the lockdown period. In March 2020, the American Academy of Ophthalmology, for example, had recommended that all elective surgery be postponed, and that all ophthalmologists should cease providing any treatment other than urgent or emergent care. (https://www.aao.org/coronavirus) Currently, there have been recommendations in certain areas for elective procedures to commence. However, there are still questions regarding how to begin elective surgeries in a safe manner that will protect our patients and healthcare providers. In addition, patient flow within clinics have also changed to observe the rules of safe distancing by spacing-out seating areas and extending gaps between appointment times to avoid overcrowding. As a result, it is certain that all units will face a significant backlog of patients after lockdown 
measures have been lifted. The continued need for social distancing, increase in patient load, coupled with limited healthcare resources will post a new set of challenges to the post COVID-19 provision of ophthalmology care.

Another key area of uncertainty is the long-term effects on vision as a result of this period of disruption in care. We do not yet know the impact of clinical cancellation and postponement of non-essential treatments. Ultimately, these are difficult decisions that physicians have to weigh up the risk of deferring sight-saving visits versus risk of COVID19 exposure which have a significant mortality rate. For example, the Royal College of Ophthalmologists recommended that patients with neovascular age-related macular degeneration should continue receiving anti-vascular endothelial growth factor 8-weekly. However, a proportion of patients may still choose not to attend either out of fear of infection or reluctance to burden the already overstretched NHS. It will be imperative that in the postlockdown period, clinical units perform audit to evaluate the impact on vision of this crisis. It will also be important to provide education to our patients about the safeguards that have been placed to protect them from being exposed to COVID-19. This may present an opportunity to explore novel technologies such as video consultation and home monitoring.

Overall, the COVID-19 pandemic is a rapidly evolving situation and it is too early to predict its eventual resolution. It is likely that many current measures will need to continue, hence a new norm may have to be established with regards to how we practise ophthalmology and medicine as a whole.

Although the current evidence related to many aspects of COVID-19 remains patchy, new data are rapidly accumulating, aided by many international collaborative efforts. Resources that enable the sharing of experiences and lessons learnt from different regions across the world, such as the monthly update by the AAO COVID resource, will help to accelerate progress in our fight against the COVID-19 crisis.

Acknowledgements Gemmy Cheung is supported by a grant from the National Medical Research Council, (Open Fund Large Collaborative grant no: NMRC/LCG/0042018). R.V. Paul Chan (Unrestricted Departmental Grant from Research to Prevent Blindness, New York, NY).

\section{Compliance with ethical standards}

Conflict of interest The authors declare that they have no conflict of interest.

Publisher's note Springer Nature remains neutral with regard to jurisdictional claims in published maps and institutional affiliations.

\section{References}

1. Ahmed F, Zviedrite N, Uzicanin A. Effectiveness of workplace social distancing measures in reducing influenza transmission: a systematic review. BMC Public Health. 2018;18:518.

2. Wilder-Smith A, Freedman DO. Isolation, quarantine, social distancing and community containment: pivotal role for old-style public health measures in the novel coronavirus (2019-nCoV) outbreak. J Travel Med. 2020;27:taaa020. https://doi.org/10.1093/ $\mathrm{jtm} / \mathrm{taaa} 020$. [Epub ahead of print].

3. Steffens I. A hundred days into the coronavirus disease (COVID-19) pandemic. Euro Surveill. 2020;25:2000550. [Epub ahead of print].

4. Leung $\mathrm{K}, \mathrm{Wu}$ JT, Liu D, Leung GM. First-wave COVID-19 transmissibility and severity in China outside Hubei after control measures, and second-wave scenario planning: a modelling impact assessment. Lancet. 2020. https://doi.org/10.1016/S0140-6736(20) 30746-7.

5. Cowling BJ, Ali ST, Ng TWY, et al. Impact assessment of nonpharmaceutical interventions against coronavirus disease 2019 and influenza in Hong Kong: an observational study. Lancet Public Health. 2020. https://doi.org/10.1016/S2468-2667(20)30090-6.

6. Chandra S, Flanagan D, Hingorani M, Lotery A, Sivaprasad S. COVID19 and ophthalmology: a brief summary of the literature. 2020. https://www.nature.com/articles/s41433-020-0956-3. 